\title{
Climate change and the diversity of its health effects
}

\author{
Paolo Vineis
}

Published online: 18 November 2009

(c) Birkhäuser Verlag, Basel/Switzerland 2009

As several contributions in this issue of International Journal of Public Health suggest (in particular that from Kjellstrom 2009), there is a wide range of potential—direct and indirect-health consequences of climate change. However, the science of such effects is still in its infancy and faces considerable challenges, as we try to suggest in our contribution (Xun et al. 2009).

Possible events attributable to climate change range from rapid and catastrophic to slow and mild. For example, IPCC does not rule out (though it is very unlikely) a cascade of events leading to Bangladesh being swept away because of rapid Himalayan glacier melting. Apart from extreme and unlikely effects, some of the direct consequences of climate change are rather obvious and can be easily perceived, such as the deaths related to heat waves in Europe in 2003. The causal pathway in these cases is clear, and does not require any particularly sophisticated epidemiological technique. Confounding is irrelevant as far as we consider the causal association between the climate event (heat wave) and its direct health consequences. But still: were all heat waves in the recent past due to what we call climate change, i.e. a man-made trend in temperature and its related epiphenomena? Was the flood in Bangladesh in 1974 the first attributable to climate change, or the last

Paolo Vineis is Chair of Environmental Epidemiology at Imperial College, London. His main interests are in the field of molecular epidemiology and gene-environment interactions. He also works on climate change with the Grantham Institute for Climate Change in London.

This paper belongs to the special issue "Climate changes health".

P. Vineis $(\square)$

Imperial College London, London, UK

e-mail: p.vineis@imperial.ac.uk not due to it? And what about the one in 1998? Clearly borders are fuzzy, and causal chains intricate.

Uncertain inferences on the causal nature of events also concern the attribution to climate change of indirect health effects, such as infectious disease outbreaks, changes in food quality and availability, water salinization and the ensuing epidemic of hypertension (Xun et al. 2009). Even wars and conflicts (like in Darfur), mass migrations and effects on mental health have been attributed to climate change. In a survey conducted among children aged between 2 and 9 in Bangladesh, Durkin et al. (1993) found post-flood changes in behaviour and bedwetting. Children were reported to have "very aggressive behaviour" after floods, with a significant increase compared to the preflood situation. A qualitative study explored the experiences of female adolescents during the 1998 floods in Bangladesh, focusing on the implications of sociocultural norms related to notions of honour, shame, purity and pollution. A number of the girls were vulnerable to sexual and mental harassment through exposure to unfamiliar environment of flood shelters and relief camps. Common mental health disorders following climate-induced displacement include anxiety, depression, post-traumatic stress disorder, irritability, sleeplessness and suicide. Moreover, conflict situations that may arise among farmers in times of climate-induced natural disasters like droughts and floods need to be addressed.

Is all of this attributable to what we call climate change? Where are the borders between the burden of events that would occur anyhow, particularly in low-income countries, even in the absence of climate change, and those attributable to the latter? How can we identify the chain of events that eventually explains the local outbreak? Again, fuzzy borders have to be acknowledged. It should be noted that the effects we have described are mainly occurring or 
foreseen in low-income countries, where disentangling new threats from the old ones is not straightforward. Also not obvious is to disentangle the complex network of interrelated determinants of single events, like the Farraka dam, shrimp farming and sea level rise in the case of water salinization in Bangladesh.

Is there a methodological specificity of the science of climate change and health (and extensively, of Global Health)? I do not think that such science is essentially different from Public Health-except for the scale-and the usual tools of epidemiologic methodology (emphasis on study design, search for bias and confounding, statistical stability of the estimates) should suffice at first glance. However, we have learnt in the investigation of the aetiology of chronic diseases such as cancer or cardiovascular diseases that simple tools and traditional causal reasoning, considering a factor at a time, can be insufficient. Though single important determinants of health—such as smoking - have been easily unveiled, the challenge posed by diseases such as breast or colon cancer requires novel approaches. Models of causality such as Bradford-Hill's, that consider single risk factors, are becoming insufficient. One of the new approaches is called "systems biology". Though we have to be sceptical towards fashions and unwarranted expectations, there might be some analogies between the development of systems biology in chronic disease epidemiology and the investigation of climate change effects (at different levels of reality, from micro to macro). Three characteristics of systems biology have been suggested as central: (a) the experimental/observational data should reflect the processes of the intact system rather than that of an isolated component; (b) data interpretation is often conducted using methods inspired by other natural sciences (e.g. physics, complex mathematical modelling); (c) an analysis of non-trivial competing explanations is conducted, and the structure of the model should reflect the underlying mechanisms in the biological system (Cedersund and Roll 2009). Similar postulates may apply to the newborn science of health effects of climate change. The analogy I propose is a challenge for epidemiologists.

Acknowledgments This research has been made possible by a contribution of the Grantham Institute for Climate Change.

\section{References}

Cedersund G, Roll J (2009) Systems biology: model based evaluation and comparison of potential explanations for given biological data. FEBS J 276(4):903-922

Durkin MS, Khan N, Davidson LL, Zaman SS, Stein ZA (1993) The effects of a natural disaster on child behavior: evidence for posttraumatic stress. Am J Public Health 83(11):1549-1553

Kjellstrom T (2009) Public health impact of global heating due to climate change: potential effects on chronic non-communicable diseases. Int J Public Health. doi:10.1007/s00038-009-0090-2

Xun WW, Khan AE, Michael E (2009) Climate change epidemiology: methodological challenges. Int J Public Health. doi: 10.1007/s00038-009-0091-1 